\title{
GCU
}

Glasgow Caledonian

University

University for the Common Good

\section{Is pupil diameter influenced by refractive error?}

Orr, Janis B.; Seidel, Dirk; Day, Mhairi; Gray, Lyle S.

Published in:

Optometry and Vision Science

DOI:

10.1097/OPX.0000000000000627

Publication date:

2015

Document Version

Author accepted manuscript

Link to publication in ResearchOnline

Citation for published version (Harvard):

Orr, JB, Seidel, D, Day, M \& Gray, LS 2015, 'Is pupil diameter influenced by refractive error?', Optometry and Vision Science, vol. 92, no. 7, pp. 834-840. https://doi.org/10.1097/OPX.0000000000000627

\section{General rights}

Copyright and moral rights for the publications made accessible in the public portal are retained by the authors and/or other copyright owners and it is a condition of accessing publications that users recognise and abide by the legal requirements associated with these rights.

Take down policy

If you believe that this document breaches copyright please view our takedown policy at https://edshare.gcu.ac.uk/id/eprint/5179 for details of how to contact us. 
Is Pupil Diameter Influenced by Refractive Error?

Janis B. Orr*, Dirk Seidel**, Mhairi Day**, and Lyle S. Gray**

*Department of Department of Life and Health Sciences, Aston University, Birmingham, United Kingdom

**Department of Life Sciences, Glasgow Caledonian University, Glasgow, United Kingdom

\section{ABSTRACT}

Purpose. To investigate the relationship between pupil diameter and refractive error and how refractive correction, target luminance, and accommodation modulate this relationship. Methods. Sixty emmetropic, myopic, and hyperopic subjects (age range, 18 to 35 years) viewed an illuminated target (luminance: 10, 100, 200, 400, 1000, 2000, and $4100 \mathrm{~cd} / \mathrm{m} 2$ ) within a Badal optical system, at 0 diopters (D) and j3 D vergence, with and without refractive correction. Refractive error was corrected using daily disposable contact lenses. Pupil diameter and accommodation were recorded continuously using a commercially available photorefractor.

Results.

Nosignificantdifferenceinpupildiameterwasfoundbetweentherefractivegroupsat0Dorj3Dtarg etvergence, in the corrected or uncorrected conditions. As expected, pupil diameter decreased with increasing luminance. Target vergence had no significant influence on pupil diameter. In the corrected condition, at $0 \mathrm{D}$ target vergence, the accom- modation response was similar in all refractive groups. At j3 D target vergence, the emmetropic and myopic groups accommodated significantly more than the hyperopic group at all luminance levels. There was no correlation between accommodation response and pupil diameter or refractive error in any refractive group. In the uncorrected condition, the accommodation response was significantly greater in the hyperopic group than in the myopic group at all luminance levels, particularly for near viewing. In the hyperopic group, 
the accommodation response was significantly correlated with re- fractive error but not pupil diameter. In the myopic group, accommodation response level was not correlated with refractive error or pupil diameter.

Conclusions. Refractive error has no influence on pupil diameter, irrespective of refractive correction or accommodative demand. This suggests that the pupil is controlled by the pupillary light reflex and is not driven by retinal blur. (Optom Vis Sci 2015;92:00Y00)

Key Words: pupil, refractive errors, myopia, accommodation, ocular, optical quality

The pupil forms the physical aperture stop of the optical system of the eye ${ }^{1}$, controlling both retinal illuminance and retinal image quality. ${ }^{2-8}$ Retinal image quality of the corrected eye is optimum at pupil sizes of approximately 3-5 mm. ${ }^{9}$ Diffraction and higher-order order aberrations cause deterioration in quality below and above, respectively, this range. ${ }^{6,7,9-12}$ When pupils are small, vision may be adversely affected by loss of retinal illumination. ${ }^{12,13}$ It has been suggested that the primary function of the pupillary light reflex is to maximise visual acuity over a wide luminance range..$^{2,3}$

There are many factors that influence pupil diameter. The diameter of the pupil decreases with increasing age ${ }^{8,14-16}$, increasing retinal illumination, $3,8,16,17$ and when changing fixation from distance to near. ${ }^{18-22}$ The primary stimulus for the pupil near response is assumed to arise through the near triad. ${ }^{18-22}$ The near triad describes the linkage between pupil, accommodation, and convergence responses. ${ }^{18-20,22}$ Previous evidence suggests that the presence of blur-driven accommodation alone is not sufficient to drive the pupil near response. ${ }^{18-20,22}$ Proximal cues such as size change or lateral or vertical displacement of an approaching object have been found to be more compelling stimuli for the pupil near response. ${ }^{18,21,22}$

The notion that myopes have larger pupils dates back to the $18^{\text {th }}$ century. ${ }^{23}$ Anecdotal clinical evidence also suggests that myopic individuals have larger pupils than hyperopic 
individuals. ${ }^{24,25}$ Hirsch and Weymouth concluded that there is a tendency for myopic subjects to have larger pupils than hyperopic and emmetropic subjects. ${ }^{26}$ However, refractive error and accommodation were not controlled and pupil measurements were taken using a ruler, and were, therefore, subjective and crude. Other studies, where accommodation was controlled adequately, have not found any systematic difference in pupil diameter between myopic and emmetropic subjects. ${ }^{16,27,28}$

Winn et al. published the only study to investigate the pupil diameter of hyperopic subjects, under controlled conditions. ${ }^{16}$ The effects of age, gender, refractive error, and iris colour on pupil diameter were investigated in emmetropic, myopic, and hyperopic subjects. Winn et al. found that the effect of age on pupil diameter was highly significant, while pupil diameter did not vary with gender, iris colour, or refractive group. ${ }^{16}$ The hyperopic group had a greater mean age than the emmetropic and myopic groups (mean age: 40.5 years, 41.5 years and 50.8 years respectively). Due to the wide age range of subjects (17-83 years) and the difference in mean age between the refractive groups, it is possible that any refractive group differences in pupil diameter could have been confounded by the effect of age. ${ }^{8,16}$

It has not yet been established whether there is a relationship between pupil diameter and refractive error in young subjects wearing refractive correction. It has been shown, using mydriasis and artificial pupils, that when uncorrected refractive error exists, visual acuity shows a pronounced dependence on pupil diameter, with the degree of dependence increasing with refractive error. ${ }^{9}$ Physiological pupil diameter in the uncorrected eye is also of interest as many moderate hyperopic individuals $(\leq+2.00 \mathrm{D})$ do not wear any refractive correction, and few pre-presbyopic hyperopes wear their full cycloplegic refractive correction. In addition, myopes often remove their spectacles to read. Systematic pupil diameter differences between refractive groups could have important implications for the level of aberration ${ }^{7,8,29,30}$ and magnitude of the depth of focus ${ }^{8,12,13,31}$ in eyes under normal viewing conditions. 
The aims of this study were to investigate the relationship between pupil diameter and refractive error in young emmetropic, myopic, and hyperopic subjects and to examine how refractive correction, target luminance, and accommodative effort modulate this relationship.

\section{Materials and Methods}

\section{Subjects}

Sixty subjects from the student population at Glasgow Caledonian University participated with informed consent in the study (see Table 1), which was conducted in accordance with the Declaration of Helsinki. The refractive error of the right eye of each subject was determined using retinoscopy under cycloplegia ( $1 \mathrm{gtt} .1 \%$ cyclopentolate hydrochloride). A univariate analysis of variance (ANOVA) was conducted, with refractive group as the between-subject factor and age as the dependent variable. There was no significant age difference between the refractive groups $\left(F_{2,60}=2.76, p=0.07\right)$. All subjects had visual acuity of at least $6 / 6$ Snellen (LogMar 0.00 ), no systemic pathology, and astigmatism $\leq 0.50 \mathrm{DC}$ in order to justify use of Mean Spherical Equivalent (MSE) correction.

All myopic and hyperopic subjects participated in 2 experimental conditions; one where the MSE cycloplegic refractive error was fully corrected with daily disposable contact lenses (1 Day Acuvue, Johnson\&Johnson) and the other where no refractive correction was worn. The order of the 2 sets of conditions was randomised. The emmetropic subjects participated in the study only once and are included in the corrected condition. Measurements were taken from the right eye and the left eye was occluded. 


\section{Methods}

The experimental set-up is shown in Figure 1. Subjects viewed a 6/12 Snellen equivalent within an illuminated field of $5.4 \times 3.6$ degrees. Target luminance was altered using neutral density (ND) filters within a slide projector. The following target luminance levels were presented: $10,100,200,400,1000,2000$ and $4100 \mathrm{~cd} . \mathrm{m}^{-2}$. Randomisation of the order of target luminance presentation was not reasonable due to the considerable recovery time that was required between targets with large differences in luminance. Thus, the targets were presented in increasing order of brightness and one minute of adaptation at each luminance level was provided before measurements were taken. ${ }^{16}$ Targets were presented at vergence levels of $O D$ and $-3 D$ in a $+5 D$ Badal optical system.

Infra-red pupillometry was performed using a photorefractor (PowerRefractor II, Plus Optix, Erlangen, Germany). This was aligned perpendicularly to the viewing system via a hot mirror (LEGB-Borofloat, Jena, Germany) that transmitted visible light (>92\%, 425-675nm) but reflected infra-red light at $45^{\circ}(>95 \%, 750-1150 \mathrm{~nm})$. Continuous recordings of pupil diameter and spherical refractive error (in the vertical meridian) of 1 minute duration were obtained from the right eye, under each viewing condition, at a sampling frequency of $25 \mathrm{~Hz}$. The PowerRefractor II measures entrance pupil diameter, the visible image of the physical aperture stop in object space which has been magnified by refraction through the cornea. ${ }^{1,32,33}$ It has been shown to accurately measure pupil diameter and refractive error when pupil diameter is greater than $3 \mathrm{~mm},{ }^{34,35}$ although the best signal-to-noise ratio is achieved when the pupil diameter is greater than $4 \mathrm{~mm} .{ }^{35}$

In the corrected condition, the refractive error data represented the accommodation response level, whereas in the uncorrected condition the accommodation response level was calculated by subtracting the spherical refractive error component of each subject from the PowerRefractor II data. 
Statistical analysis for the pupil diameter and accommodation data was performed using a Repeated Measures ANOVA with refractive group (emmetropic/myopic/hyperopic) and target vergence $(0 D /-3 D)$ as the between subjects factors, and target luminance $(10,100$, $200,400,1000,2000$ and $4100 \mathrm{~cd} . \mathrm{m}^{-2}$ ) as the within subject variable. We used the $95 \%$ confidence limits of the mean. If a significant functional effect was identified in the main ANOVA, the data were further analysed using Bonferroni post-hoc tests. These are particularly conservative tests providing protection over type I error.

\section{Results}

\section{Pupil Diameter}

Figure 2 shows the mean pupil diameter in the emmetropic, myopic and hyperopic groups, at the $0 \mathrm{D}$ and $-3 \mathrm{D}$ target vergence levels, in the corrected and uncorrected conditions.

In the corrected condition, pupil diameter decreased significantly with increasing target luminance (Repeated Measures ANOVA $F_{6,114}=385.79, p<0.001$ ). Post-hoc tests showed that this occurred at $0 D(p<0.001)$ and $-3 D(p<0.001)$ target vergence. Pupil diameter was not significantly influenced by refractive group (Repeated Measures ANOVA $F_{2,114}=0.09$, $p=0.91)$. There was no significant difference in pupil diameter between the $0 D$ and $-3 D$ target vergence levels (Repeated Measures ANOVA $F_{1,114}=3.40, p=0.68$ ). However, when the individual difference in pupil diameter between $0 D$ and $-3 D$ target vergence was calculated, this was significantly different from zero (Repeated Measures ANOVA $\left.F_{6,46}=2.58, p=0.02\right)$.

In the uncorrected condition, pupil diameter decreased significantly with increasing target luminance (Repeated Measures ANOVA $F_{6,85}=247.22, p<0.001$ ). Post-hoc tests showed that this occurred at $0 D(p<0.001)$ and $-3 D(p<0.001)$ target vergence. Pupil diameter was 
not significantly influenced by refractive group (Repeated Measures ANOVA $F_{2,85}=0.62$, $p=0.54)$. There was a significant difference in pupil diameter between the $O D$ and $-3 D$ target vergence levels (Repeated Measures ANOVA $F_{1,85}=4.46, p=0.04$ ). However, this was not influenced by refractive group (Repeated Measures ANOVA $F_{1,85}=0.57, p=0.45$ ).

\section{Accommodation Response}

Figure 3 shows the mean accommodation response level in the corrected and the uncorrected conditions.

In the corrected condition, there was a significant difference in accommodation response between the refractive groups (Repeated Measures ANOVA $F_{2,69}=6.51, p=0.003$ ), which was significantly influenced by target vergence (Repeated Measures ANOVA $F_{2,69}=5.75$, $p=0.005)$. At $O D$ target vergence, post-hoc tests showed that there was no difference in accommodation response between the refractive groups $(p=0.99)$. At $-3 D$ target vergence, post-hoc tests showed that there was a significant difference in accommodation response between the refractive groups $(p<0.001)$. Post-hoc tests showed that accommodation response was significantly greater in the emmetropic $(p<0.001)$ and the myopic $(p<0.001)$ groups than the hyperopic group, and this was the case at all target luminances $(p<0.001$ for all comparisons). There was no difference in accommodation response between the emmetropic and myopic groups $(p=0.91)$.

In the uncorrected condition, there was a significant difference in accommodation response between the refractive groups (Repeated Measures ANOVA F A $_{1,64}=88.23$, $p<0.001$ ), which was significantly influenced by target vergence (Repeated Measures ANOVA $\left.F_{2,64}=5.13, p=0.03\right)$. Pairwise comparisons showed that the accommodation response was significantly greater in the hyperopic group than the myopic group at all 
luminance levels in both the $0 D(p<0.012$ for all comparisons) and $-3 D(p<0.001$ for all comparisons) target vergence conditions.

\section{Correlation between Accommodation Response and Pupil Diameter}

Accommodation response level was not significantly correlated with pupil diameter at OD (emmetropic group: Pearson correlation $\mathrm{R}=0.52, \mathrm{p}=0.07$; myopic group: Pearson correlation $R=-0.23, p=0.31$; hyperopic group: Pearson correlation $R=0.33, p=0.20$ ) or $-3 D$ (emmetropic group: Pearson correlation $\mathrm{R}=0.09, \mathrm{p}=0.70$; myopic group: Pearson correlation $\mathrm{R}=0.24, \mathrm{p}=0.27$; hyperopic group: Pearson correlation $\mathrm{R}=0.01, \mathrm{p}=0.97$ ) target vergence, in the corrected condition. Neither was accommodation response level correlated with pupil diameter at $\mathrm{OD}$ (myopic group: Pearson correlation $\mathrm{R}=0.47, \mathrm{p}=0.06$; hyperopic group: Pearson correlation $\mathrm{R}=-0.56, \mathrm{p}=0.06$ ) or $-3 \mathrm{D}$ (myopic group: Pearson correlation $\mathrm{R}=0.28, \mathrm{p}=0.24$; hyperopic group: Pearson correlation $\mathrm{R}=0.02, \mathrm{p}=0.94$ ) target vergence, in the uncorrected condition.

\section{Correlation between Accommodation Response and Refractive Error}

In the corrected condition, accommodation response level was not significantly correlated with refractive error at $\mathrm{OD}$ (emmetropic group: Pearson correlation $\mathrm{R}=0.30, p=0.20$; myopic group: Pearson correlation $R=0.20, p=0.09$; hyperopic group: Pearson correlation $R=0.09$, $p=0.73$ ) or $-3 D$ (emmetropic group: Pearson correlation $R=0.03, p=0.91$ : myopic group:Pearson correlation $\mathrm{R}=0.15, \mathrm{p}=0.68$; hyperopic group: Pearson correlation $\mathrm{R}=0.21$, $\mathrm{p}=0.42$ ) target vergence.

In the uncorrected condition, accommodation response level was significantly correlated with refractive error in the hyperopic group at $\mathrm{OD}$ (Pearson correlation $\mathrm{R}=0.60, p=0.04$ ) and $-3 \mathrm{D}$ (Pearson correlation $\mathrm{R}=0.72, \mathrm{p}=0.004$ ) target vergence. There was no such correlation 
in the myopic group (OD target vergence: Pearson correlation $R=0.09, p=0.46 ;-3 D$ target vergence: Pearson correlation $\mathrm{R}=0.28, \mathrm{p}=0.36$ ).

\section{Discussion}

The main aims of this study were to establish whether there is any systematic variation in pupil diameter with refractive error in young emmetropic, myopic and hyperopic subjects, and to examine how refractive correction, target luminance, and accommodative effort modulate this relationship.

Winn et al. published the only prior study to investigate the pupil diameter of hyperopic subjects, under controlled experimental conditions, where the effects of age, gender, refractive error, and iris colour upon pupil diameter were investigated, in subjects ranging in age from 17-83 years. ${ }^{16}$ Refractive error was fully corrected and the target was presented at infinity (OD target vergence). Winn et al. found that age was the only factor to significantly influence pupil diameter. ${ }^{16}$ The results of the present study both agree with the refractive error findings of Winn et al. ${ }^{16}$ and extend previous work by confirming that pupil diameter does not vary between refractive groups, when the confounding factor of age is removed. The present study has the added uniqueness of demonstrating that this finding is independent of target vergence. The results are in agreement with other studies, ${ }^{21,22}$ which demonstrated, in emmetropic subjects, that the presence of blur-driven accommodation alone is not sufficient to drive the pupil near response. The present study extends previous work by also including myopic and hyperopic subjects.

Hirsch and Weymouth reported larger pupils in myopes, compared to emmetropes and hyperopes, when refractive error was uncorrected. ${ }^{26}$ However, the accommodation response was not measured or controlled, the target luminance was not standardised and the pupil diameter was measured subjectively. ${ }^{26}$ The present study is the first to measure 
pupil diameter without refractive error correction, under controlled experimental conditions, and demonstrates that hyperopic individuals have similar pupil diameters to emmetropic and myopic individuals, both when corrected and uncorrected, for distance and near viewing. This is surprising given that the hyperopic group exerted greater levels accommodation than the emmetropic and myopic group, when no refractive error correction was worn, at $0 D$ and $-3 D$ target vergence. There was no correlation between pupil diameter and accommodation response in any condition. These findings suggest that accommodation is not a major contributor to pupil diameter.

In the corrected condition, at OD target vergence, the mean accommodation response was $<0.50 \mathrm{D}$ in all refractive groups, which was expected given that subjects were fully corrected and under no accommodative demand. However, when an accommodative demand was present, the hyperopic group accommodated less than the emmetropic and myopic groups, who accommodated by a similar amount. This finding was unexpected but could be due to the fact that the hyperopes were wearing their full cycloplegic correction and were, therefore, functionally overcorrected (as young hyperopes are accustomed to being under-corrected and will not normally tolerate their full correction). Had the hyperopic group been wearing their habitual refractive correction, it is expected that they would have accommodated by a similar amount to the emmetropic and myopic groups (although, unfortunately, this was not tested). It is worth noting that all of the refractive groups demonstrated accommodative lag.

In the uncorrected condition, at OD target vergence, the myopic group did not accommodate more than $0.50 \mathrm{D}$. This was expected as the target would appear blurred to these myopic individuals and any accommodation response would only increase the level of blur. At -3D target vergence, the myopic group only accommodated by an average of $\sim 1.00 \mathrm{D}$. This is due to the fact that the (near) target would be relatively clear to many of the myopic subjects as it was positioned at their far point and a full accommodative response was not required. Despite taking their refractive error into account, by subtracting 
the spherical refractive error component of each subject from the PowerRefractor II refractive error data, the hyperopic group over-accommodated at both the $0 D$ and $-3 D$ vergence levels $(\sim 2.50 \mathrm{D}$ and $\sim 4.50 \mathrm{D}$ respectively). This finding was unexpected and the reasons for this are unclear. We suggest that this reduction in accuracy of the accommodation response was caused by the additional accommodative demand placed on the hyperopic group by their uncorrected refractive error. Alternatively, it is possible that cyclopentolate $(1 \%)$ did not reveal their full refractive error which could explain this exaggerated accommodation response.

The results of the present study suggest that refractive error has no influence upon pupil diameter, irrespective of refractive correction or accommodative demand. This suggests that the pupil is controlled by the pupillary light reflex and is not driven by retinal blur.

\section{Acknowledgements}

This research received no specific grant from any funding agency in the public, commercial, or not-for-profit sectors.

The authors would like to acknowledge Professor Niall Strang for his comments on an early version of this manuscript.

\section{Disclosure Statement}

The authors report no conflicts of interest and have no proprietary interest in any of the materials mentioned in this article.

Elements of this manuscript have been presented as: a paper at ARVO, May 2010, Fort Lauderdale, Florida, USA; a poster at the 13th International Myopia Conference, July 
2010, University of Tuebingen, Germany; and a poster at ARVO, May 2011, Fort

Lauderdale, Florida, USA.

\section{References}

1. Bennett AG, Rabbetts RB. Clinical Visual Optics, 3rd ed. Edinburgh: ButterworthHeinemann; 1998.

2. Campbell FW, Gregory AH. Effect of size of pupil on visual acuity. Nature 1960;187:1121-3.

3. Laughlin SB. Retinal information capacity and the function of the pupil. Ophthalmic Physiol Opt 1992;12:161-4.

4. Woodhouse JM, Campbell FW. The role of the pupil light reflex in aiding adaptation to the dark. Vision Res 1975;15:649-53.

5. Sakai H, Hirata Y, Usui S. Relationship between residual aberration and lightadapted pupil size. Optom Vis Sci 2007;84:517-21.

6. Liang J, Williams DR. Aberrations and retinal image quality of the normal human eye. J Opt Soc Am (A) 1997;14:2873-83.

7. Campbell FW, Gubisch RW. The effect of chromatic aberration on visual acuity. J Physiol 1967;192:345-58.

8. Watson AB, Yellott JI. A unified formula for light-adapted pupil size. J Vis 2012;12:1-16.

9. Atchison DA, Smith G, Efron N. The effect of pupil size on visual acuity in uncorrected and corrected myopia. Am J Optom Physiol Opt 1979;56:315-23.

10. Donnelly WJ, 3rd, Roorda A. Optimal pupil size in the human eye for axial resolution. J Opt Soc Am (A) 2003;20:2010-5.

11. Applegate RA, Donnelly WJ, 3rd, Marsack JD, Koenig DE, Pesudovs K. Threedimensional relationship between high-order root-mean-square wavefront error, pupil diameter, and aging. J Opt Soc Am (A) 2007;24:578-87.

12. Campbell FW, Gubisch RW. Optical quality of the human eye. J Physiol 1966;186:558-78.

13. Donnelly WJ, 3rd, Roorda A. Optimal pupil size in the human eye for axial resolution. J Opt Soc Am (A) 2003;20:2010-5.

14. Kadlecova V, Peleska M, Vasko A. Dependence on age of the diameter of the pupil in the dark. Nature 1958;182:1520-1.

15. Schaeffel F, Wilhelm H, Zrenner E. Inter-individual variability in the dynamics of natural accommodation in humans: relation to age and refractive errors. J Physiol 1993;461:301-20.

16. Winn B, Whitaker D, Elliott DB, Phillips NJ. Factors affecting light-adapted pupil size in normal human subjects. Invest Ophthalmol Vis Sci 1994;35:1132-7.

17. Loewenfield I. The Pupil. Detroit: Wayne State University Press/Ames Iowa State University Press; 1993.

18. Kasthurirangan S, Glasser A. Characteristics of pupil responses during far-to-near and near-to-far accommodation. Ophthalmic Physiol Opt 2005;25:328-39. 
19. Marg E, Morgan MW, Jr. The pupillary near reflex; the relation of pupillary diameter to accommodation and the various components of convergence. Am J Optom Arch Am Acad Optom 1949;26:183-98.

20. Marg E, Morgan MW, Jr. Further investigation of the pupillary near reflex; the effect of accommodation, fusional convergence and the proximity factor on pupillary diameter. Am J Optom Arch Am Acad Optom 1950;27:217-25.

21. Stakenburg M. Accommodation without pupillary constriction. Vision Res 1991;31:267-73.

22. Phillips NJ, Winn B, Gilmartin B. Absence of pupil response to blur-driven accommodation. Vision Res 1992;32:1775-9.

23. Porterfield W. Treatise on the Eye, the Manner and Phaenomena of Vision. London: Miller; 1759.

24. Alexandridis E. The Pupil. Heidelberg: Springer-Verlag; 1985.

25. Zinn K. The Pupil. New York: Charles C Thomas; 1972.

26. Hirsch MJ, Weymouth FW. Pupil size in ametropia. J Appl Physiol 1949;1:646-8.

27. Jones R. Do women and myopes have larger pupils? Invest Ophthalmol Vis Sci 1990;31:1413-5.

28. Charman WN, Radhakrishnan H. Accommodation, pupil diameter and myopia. Ophthalmic Physiol Opt 2009;29:72-9.

29. Liang J, Williams DR. Aberrations and retinal image quality of the normal human eye. J Opt Soc Am (A) 1997;14:2873-83.

30. Applegate RA, Donnelly WJ, 3rd, Marsack JD, Koenig DE, Pesudovs K. Threedimensional relationship between high-order root-mean-square wavefront error, pupil diameter, and aging. J Opt Soc Am (A) 2007;24:578-87.

31. Ward PA, Charman WN. Effect of pupil size on steady state accommodation. Vision Res 1985;25:1317-26.

32. Tunnacliffe AH. Introduction to Visual Optics, 4th ed. London: Association of British Dispensing Opticians; 1993.

33. Atchison DA, Smith G. Optics of the Human Eye. Melbourne, Australia:

Butterworth Heinemann; 2002.

34. Wolffsohn JS, Hunt OA, Gilmartin B. Continuous measurement of accommodation in human factor applications. Ophthalmic Physiol Opt 2002;22:380-4.

35. Allen PM, Radhakrishnan H, O'Leary DJ. Repeatability and validity of the PowerRefractor and the Nidek AR600-A in an adult population with healthy eyes. Optom Vis Sci 2003;80:245-51.

\section{Figure Legends}

Figure 1: Experimental set-up (not to scale)

Figure 2: Pupil diameter and target luminance in the emmetropic (E), myopic (M) and the hyperopic (H) groups, at OD and -3D target vergence when refractive error was corrected and not corrected. Error bars are standard deviations

Figure 3: The mean accommodation response levels for all refractive groups in the emmetropic (E), myopic (M) and hyperopic (H) groups in: a) the corrected condition at OD target vergence; b) the 
corrected condition at 3D target vergence; c) the uncorrected condition at OD target vergence; d) the uncorrected condition at 3D target vergence. In the uncorrected condition, the accommodation response level was calculated by subtracting the spherical refractive error component of each subject from the PowerRefractor II data. Error bars are standard deviations 
Figure 1

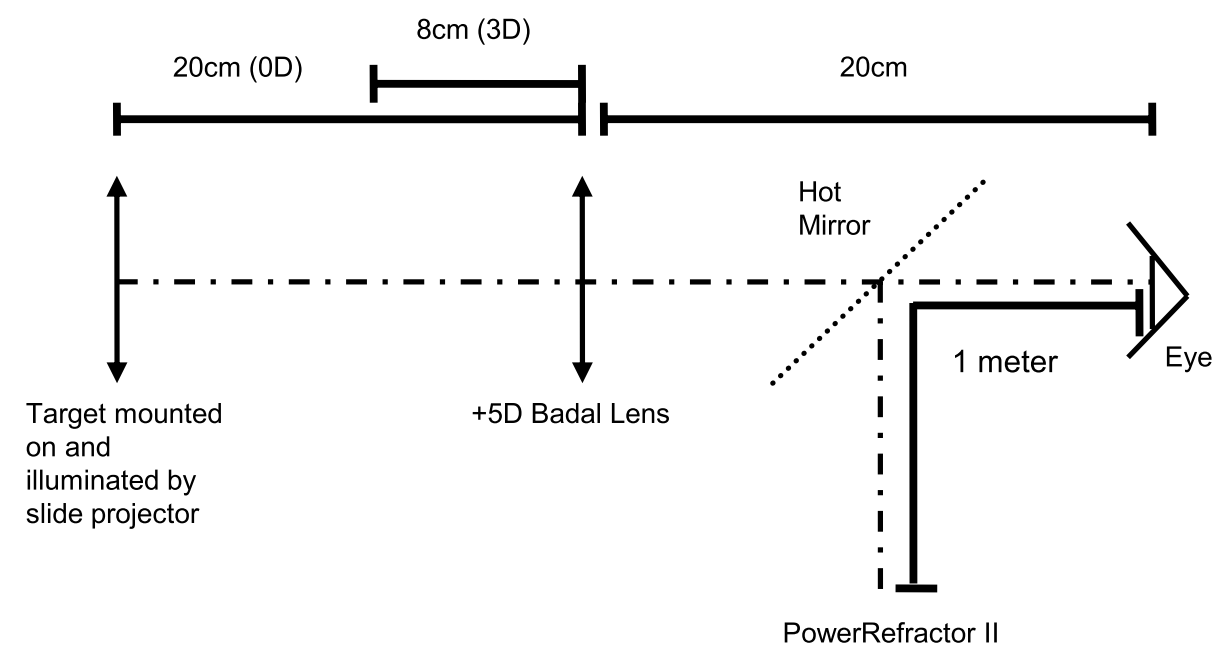


Figure 2

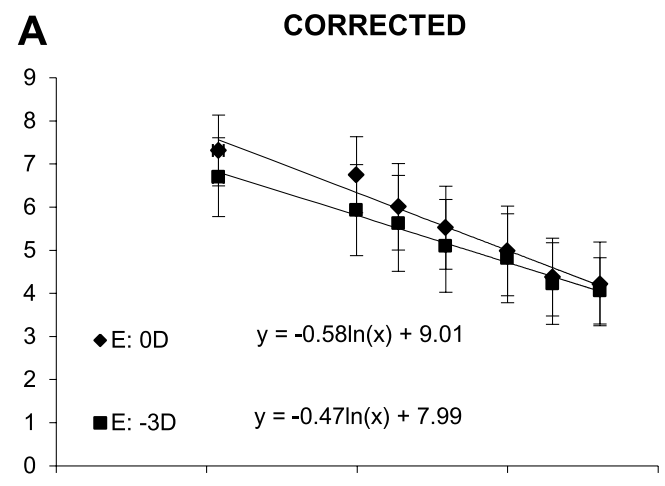

B

D
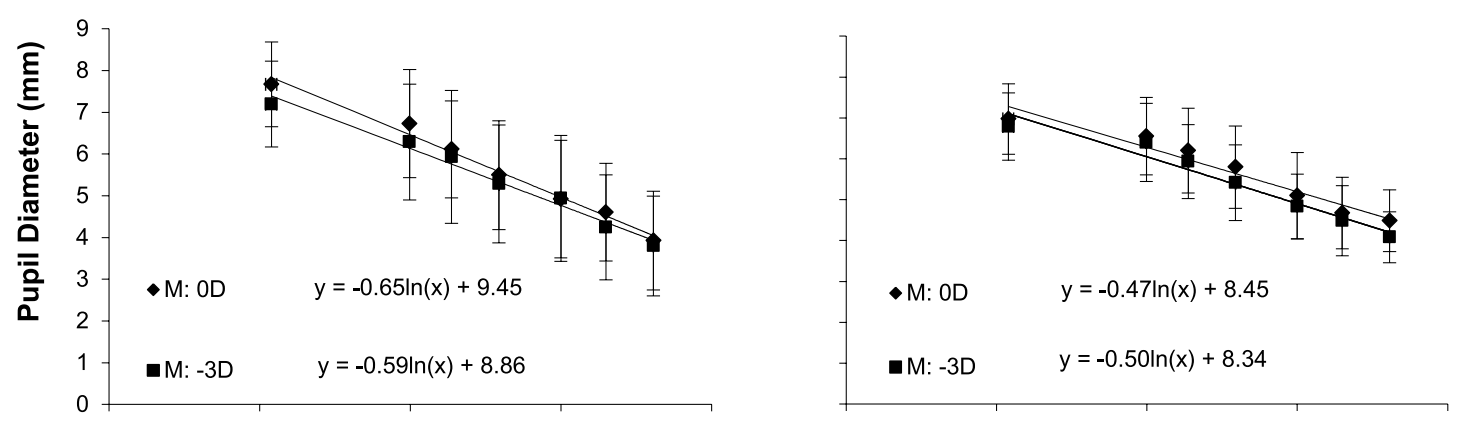

C

E

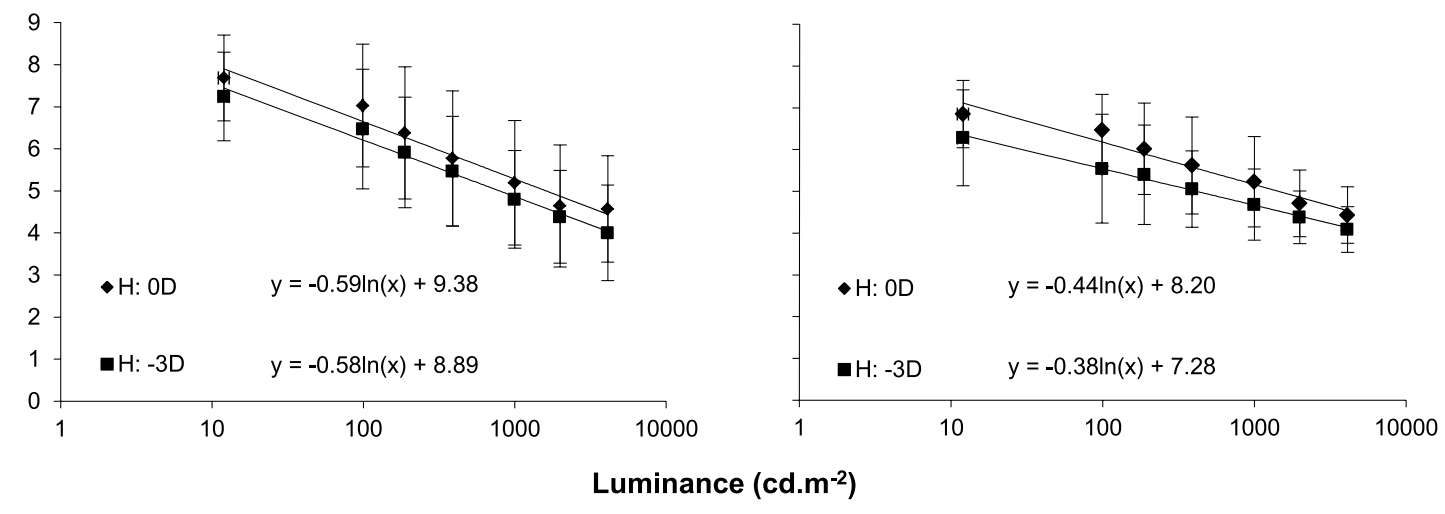


Figure 3

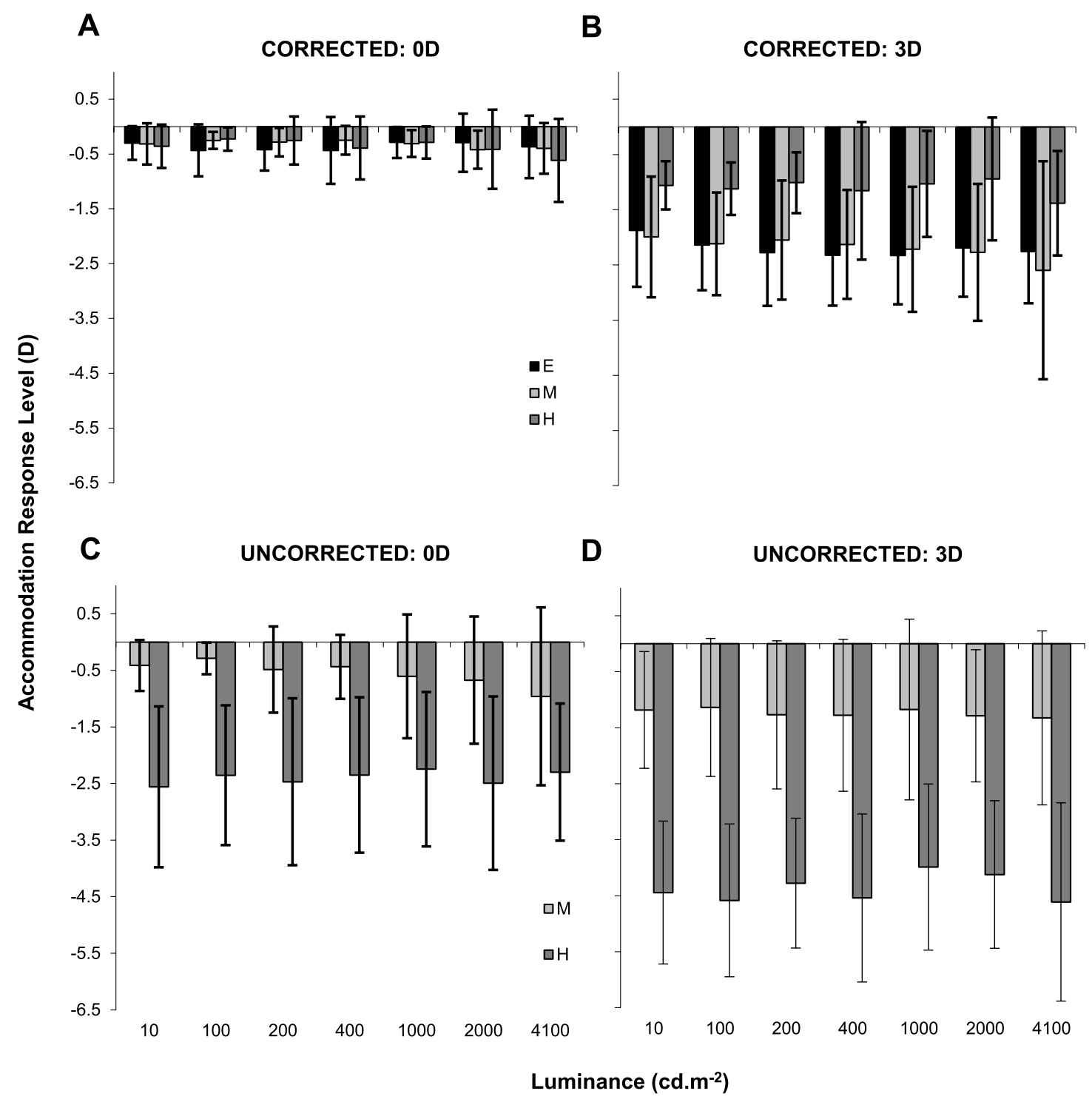

